\title{
MUTUAL INTERFERENCE BETWEEN PREDATORS CAN GIVE RISE TO TURING SPATIAL PATTERNS
}

\author{
David Alonso, ${ }^{1,2,3}$ Frederic Bartumeus, ${ }^{1}$ And Jordi Catalan ${ }^{1}$ \\ ${ }^{1}$ Department of Ecology, Biology Faculty, University of Barcelona, Diagonal 645, 08028 Barcelona, Spain \\ ${ }^{2}$ Complex Systems Research Group, Polytechnic University of Catalunya, Campus Nord B4, 08034 Barcelona, Spain
}

Abstract. The study of spatial patterns in the distribution of organisms is a central issue in ecology. Here we address the question of whether predator-prey interactions can induce nonuniform distributions. We study how diffusion affects the stability of predatorprey coexistence equilibria and show a new difference between ratio- and prey-dependent models. Recently, Peter Abrams and Lev Ginzburg reviewed the controversial issue of what kind of function better describes the rate of prey consumption by an average predator, the so-called "predator functional response." Here, we focus on reaction-diffusion predatorprey models with and without predator dependence in the functional response. We show that classical prey-dependent models cannot give rise to spatial structures through diffusiondriven instabilities; however, predator-dependent models with the same degree of complexity can. The origin of predator dependence in the rate of prey consumption is the mutual interference between predators. Therefore, we show that this mechanism can generate patchiness in a homogeneous environment under certain conditions of trophic interaction and predator-prey relative diffusion.

Key words: Beddington's functional response; functional response, ratio-dependent; linear stability analysis; patchiness in a homogeneous environment; predators, mutual interference effects; predatorprey models; prey dependence vs. ratio dependence; reaction-diffusion models; Turing patterns.

\section{INTRODUCTION}

The study of spatial patterns in the distribution of organisms is a central issue in ecology. The origin of these patterns has been commonly attributed to two sorts of sources (Levin 1992). First, a heterogeneous distribution of abiotic factors and, second, underlying mechanisms at the level of individuals. Patterns generated in abiotically homogeneous environments are particularly interesting because they require an explanation based on the individual behavior of organisms. They are commonly called "emergent patterns," because they emerge through interactions in spatial scales that are much larger than the characteristic scale of individuals. Within this context, we may ask to what extent predator-prey interaction can induce nonuniform distributions.

During the past decade a lot of studies have been devoted to the understanding of the consequences of assuming either a prey-dependent or a ratio-dependent predator functional response in predator-prey models (Ginzburg and Akçakaya 1992, Abrams 1994, Akçakaya et al. 1995). Although it has been recognized that precise prey dependence or ratio dependence will likely both be rare in nature (Abrams and Ginzburg 2000), the sort of functional response assumed strongly influences dynamic stability, the way the predator-prey system reacts to environmental influences, and how those

Manuscript received 6 November 2000; revised 19 March 2001; accepted 25 April 2001; final version received 31 May 2001.

${ }^{3}$ E-mail: david@complex.upc.es perturbations spread out through the food web. Here we show that there is a new feature that clearly depends on the sort of predation model assumed: the rise of spatial patterns through diffusion instability (Turing structures).

Turing (1952) showed how the coupling of reaction and diffusion can induce pattern formation. Since then, the mechanism responsible for the spontaneous generation of spatial patterns through biological or chemical interactions has been called "diffusion instability," and the resulting stationary inhomogeneities have been referred to as "Turing structures." Initially, Turing's ideas were applied in the context of developmental biology, but thereafter Lotka-Volterra-like formulations (Volterra 1926) in ecology were also considered under the conceptual framework of reactiondiffusion systems (Segel and Jackson 1972, Levin and Segel 1976, Rosen 1977, Mimura and Murray 1978, Okubo 1980, Conway 1984, Murray 1989).

In this paper we show that mutual predator interference is a behavioral mechanism that can be responsible for patchiness through an interplay between local population processes and diffusion. In spite of the great number of studies that have been devoted to the search for processes on small scales that can induce patterns on much larger scales, mutual interference between predators has not been previously reported. Using linear stability analysis we demonstrate that adding a diffusion term to a simple general model for predator-prey dynamics with predator-dependent functional response (Arditi and Ginzburg 1989, Ginz- 
burg and Akçakaya 1992, Akçakaya et al. 1995) a reaction-diffusion system that can develop diffusiondriven instabilities is obtained. On the contrary, if the functional response depends only on prey density, diffusion instabilities are not possible. Finally, we discuss how Turing structures are expected to arise in natural systems.

\section{A Predator-Prey Model with Diffusion}

Classical predator-prey models are written as a system of first-order differential equations. A general model can be written as follows (Abrams and Ginzburg 2000):

$$
\begin{aligned}
& \frac{d N}{d t}=f(N) N-g(N, P) P \\
& \frac{d P}{d t}=h[g(N, P), P] P
\end{aligned}
$$

where $N$ and $P$ are prey and predator densities, respectively, $f(N)$ is the prey growth rate, $g(N, P)$ is the functional response, e.g., the prey consumption rate by an average single predator, and $h[g(N, P), P]$ is the per capita growth rate of predators (also known as the "predator numerical response"), which obviously increases with the prey consumption rate, $g(N, P)$, and can be influenced by the predator density. The most widely accepted assumption (Arditi and Ginzburg 1989) for the numerical response is the linear one:

$$
h[g(N, P), P]=\operatorname{eg}(N, P)-\mu
$$

where $\mu$ is a per capita predator death rate and $e$ is the conversion efficiency of food into offspring. Ginzburg (1998:325) calls Eq. 3 "linear conversion rule," i.e., the relationship between the prey consumption rate and the per capita growth rate of predators. Strong empirical evidence for the validity of this conversion rule has been reported (Beddington et al. 1976). Introducing this common numerical response in predator Eq. 2, and adding diffusion terms in one dimension, the following reaction-diffusion system is obtained as a model for predator-prey trophic interaction:

$$
\begin{aligned}
& \frac{d N}{d t}=f(N) N-g(N, P) P+D_{N} \frac{\partial^{2} N}{\partial x^{2}} \\
& \frac{d P}{d t}=e g(N, P) P-\mu P+D_{P} \frac{\partial^{2} P}{\partial x^{2}}
\end{aligned}
$$

where $D_{N}$ and $D_{P}$ are, respectively, prey and predator diffusion coefficients, prey growth rate and the functional response are expressed in general terms, and predator death rate is assumed to be constant. The movement of organisms is modeled in a very simple way through diffusion coefficients, which assume that both prey and predator individuals don't exhibit any kind of coordinated or cooperative motion behavior but just random movements.

\section{Predator-Dependent Models: Presence of TURING STRUCTURES}

\section{The predator functional response}

The predator functional response is the essential link describing a predator-prey model. Beddington (1975) was the first to call attention to the effect of mutual interference between predators on searching efficiency and proposed the following formulation for the rate of prey consumption for an average predator:

$$
g(N, P)=\frac{b N}{B+k P+N}
$$

where $b$ is a maximum consumption rate, $k$ is a predator interference parameter, and $B$ a saturation constant, and $N, P$, and $g(N, P)$ are as in Eqs. 1 and 2.

In fact, Beddington's formulation can be considered a quite general functional response. First, Beddington's equation has been formally derived from general mechanistic assumptions (Ruxton et al. 1992, Huisman and de Boer 1997). Second, most plausible functional responses suggested in the literature-i.e, Holling II, which is prey dependent (Holling 1959, 1966), and the one proposed by Arditi and Ginzburg (1989), which is ratio-dependent-are particular cases of Eq. 6 (Ruxton et al. 1992). And, third, although not proved from mechanistic assumptions, Beddington's equation could even include the case where predators benefit from cofeeding $(k<0)$.

Some derivations (Ruxton et al. 1992, Huisman and de Boer 1997) have shed light on the meaning of the parameters occurring in Beddington's functional response. Both Ruxton et al. (1992) and Huisman and de Boer (1997) assume that predators and prey are uniformly distributed within a fixed volume, and that prey are attacked by randomly searching predators. Ruxton et al. (1992) consider that predators spend time searching and handling prey but also waste time in useless encounters with other predators. In contrast, Huisman and de Boer (1997) assume a pre-handling interaction complex between a prey and a predator. Predator attacks are not instantaneous. While a predator focuses on a single prey, no other predator will attempt to catch that prey. This is the reason why predator interference emerges. It is not a direct interference as in the Ruxton et al. (1992) model. In any case, parameter $k$ is clearly a measure of predator interference in Eq. 6.

If predators do not waste time interacting with one another (Ruxton et al. 1992) or if their attacks are always successful and instantaneous then a Holling type II functional response is obtained:

$$
g(N)=\frac{b N}{B+N} .
$$

On the other hand, taking $B=0$ in Eq. 6, the ratiodependent functional response is obtained: 


$$
g(N, P)=\frac{b N}{k P+N} .
$$

According to Ruxton et al. (1992) this limit case should be interpreted as a situation where predators are able to explore large volumes per unit time, although other interpretations have also been suggested (Huisman and de Boer 1997). The per capita rate of prey consumption in Eq. 8 depends on the ratio of prey to predator. Since Arditi and Ginzburg (1989) compared this particular case of predator dependency with general prey-dependent models, a lot of effort has been devoted to understanding the divergent consequences of assuming one of the two idealized forms of functional response. Although ecologists are aware that both functional responses are ideal extreme approximations to reality, the prey-dependent vs. ratio-dependent debate remains (Abrams and Ginzburg 2000). Our present work adds a new feature to the different expectations from the two types of functional response.

\section{Stability analysis of the ratio-dependent model}

The predator-prey model given by the reaction-diffusion system (Eqs. 4 and 5) with prey-dependent functional response cannot give rise to Turing structures (see the Appendix). Below we demonstrate that in contrast to that general prey-dependent formulation, an analogous ratio-dependent model does give rise to Turing structures.

For the stability analysis of the ratio-dependent prey model we assume self-damping prey growth rate, $f(N)$ $=r\left(1-N / N_{\max }\right)$, the linear conversion rule (Eq. 3), and a ratio-dependent functional response (Eq. 8). The resulting model is

$$
\begin{aligned}
& \frac{\partial N}{\partial T}=r\left(1-\frac{N}{N_{\max }}\right) N-\frac{b N P}{k P+N}+D_{N} \frac{\partial^{2} N}{\partial X^{2}} \\
& \frac{\partial P}{\partial T}=e \frac{b N P}{k P+N}-\mu P+D_{P} \frac{\partial^{2} P}{\partial X^{2}} .
\end{aligned}
$$

We introduce the following dimensionless variables, $n=N / \nu, p=P / \pi, t=T / \tau$, and $x=X / \lambda$, where $\nu=$ $N_{\text {max }}, \pi=N_{\max } / k, \tau=1 / r$, and $\lambda=\sqrt{D_{N} / r}$ are the units in which dimensional variables will be scaled. Thus, in terms of these dimensionless variables the model is simplified to

$$
\begin{aligned}
& \frac{\partial n}{\partial t}=(1-n) n-\frac{\beta n p}{p+n}+\frac{\partial^{2} n}{\partial x^{2}} \\
& \frac{\partial p}{\partial t}=\varepsilon \beta \frac{n p}{p+n}-\eta p+d \frac{\partial^{2} p}{\partial x^{2}}
\end{aligned}
$$

where four dimensionless new parameters naturally arise:

$$
\beta=\frac{1}{k} \frac{b}{r} \quad \eta=\frac{\mu}{r} \quad \varepsilon=e k \quad d=\frac{D_{P}}{D_{N}} .
$$

Defining a new dimensionless parameter, $\Delta=\varepsilon \beta / \eta$, the system has a homogeneous coexistence point, $\left(n^{*}\right.$, $\left.p^{*}\right)$, where $n^{*}=1-(\eta / \varepsilon)(\Delta-1)$ and $p^{*}=(\Delta-$ 1) $n^{*}$. So, in order to have a feasible coexistence point, two conditions are needed: $\eta>\varepsilon(\beta-1)$ and $\eta<\varepsilon \beta$.

Furthermore, the three conditions for diffusion instability to arise (see the Appendix; see also Okubo 1980, Murray 1989) can be summarized as follows:

$$
\begin{aligned}
& a_{11}+a_{22}<0 \\
& a_{11} a_{22}-a_{21} a_{12}>0 \\
& d a_{11}+a_{22}>2 \sqrt{d} \sqrt{\operatorname{det}\left(\mathbf{J}^{*}\right)}
\end{aligned}
$$

where $\operatorname{det}\left(\mathbf{J}^{*}\right)$ is the determinant of the community matrix for the system described by Eqs. 11 and 12, evaluated at the coexistence point, $\left(n^{*}, p^{*}\right)$. As usual, $a_{11}$, $a_{12}, a_{21}$, and $a_{22}$ are the corresponding entries of the community matrix, and $d$ is the relative diffusion, $d=$ $D_{P} / D_{N}$.

Using the standard definition of the elements of the community matrix (Okubo 1980), and defining four auxiliary functions,

$$
\begin{aligned}
g(\eta) & \equiv(\varepsilon-1) \eta^{2}-\varepsilon^{2} \beta \eta+\beta \varepsilon^{2}(\beta-1) \\
f(\eta) & \equiv \eta^{2}(\varepsilon \beta-\eta) \varepsilon^{2} \beta(\eta-\varepsilon(\beta-1)) \\
F(\eta) & \equiv \sqrt{f(\eta)} \\
G(\eta, d) & \equiv \frac{d(\beta-1) \varepsilon^{2} \beta}{2 \sqrt{d}}-\frac{\varepsilon^{2} \beta}{2 \sqrt{d}} \eta+\frac{(\varepsilon-d)}{2 \sqrt{d}} \eta^{2}
\end{aligned}
$$

the last three conditions (Inequalities 14-16) can be rewritten as

$$
\begin{aligned}
g(\eta) & <0 \\
f(\eta) & >0 \\
G(\eta, d) & >F(\eta) .
\end{aligned}
$$

In the parameter domain where feasible coexistence points exist, the simultaneous fulfillment of the last three conditions implies that uniform stable points will be altered by nonuniform small perturbations. In other words, in those situations, spatially uniform steady-state predator-prey coexistence is no longer stable. Small random fluctuations will be strongly amplified by diffusion, leading to nonuniform population distributions.

The formation of the spatial pattern through diffusion, in the long run, has been addressed numerically. To illustrate the results, several numerical integrations of the reaction-diffusion system (Eqs. 11-12) have been performed in one and two dimensions. As an example, the formation of a regular macroscopic twodimensional spatial pattern is shown in Fig. 1.

\section{Determining the Turing Space}

In order to find the region of the parameter space where diffusion instability takes place, the conditions given by Inequalities $21-23$ must be considered again. The positive coexistence conditions are just those required to have a positive determinant, i.e., 

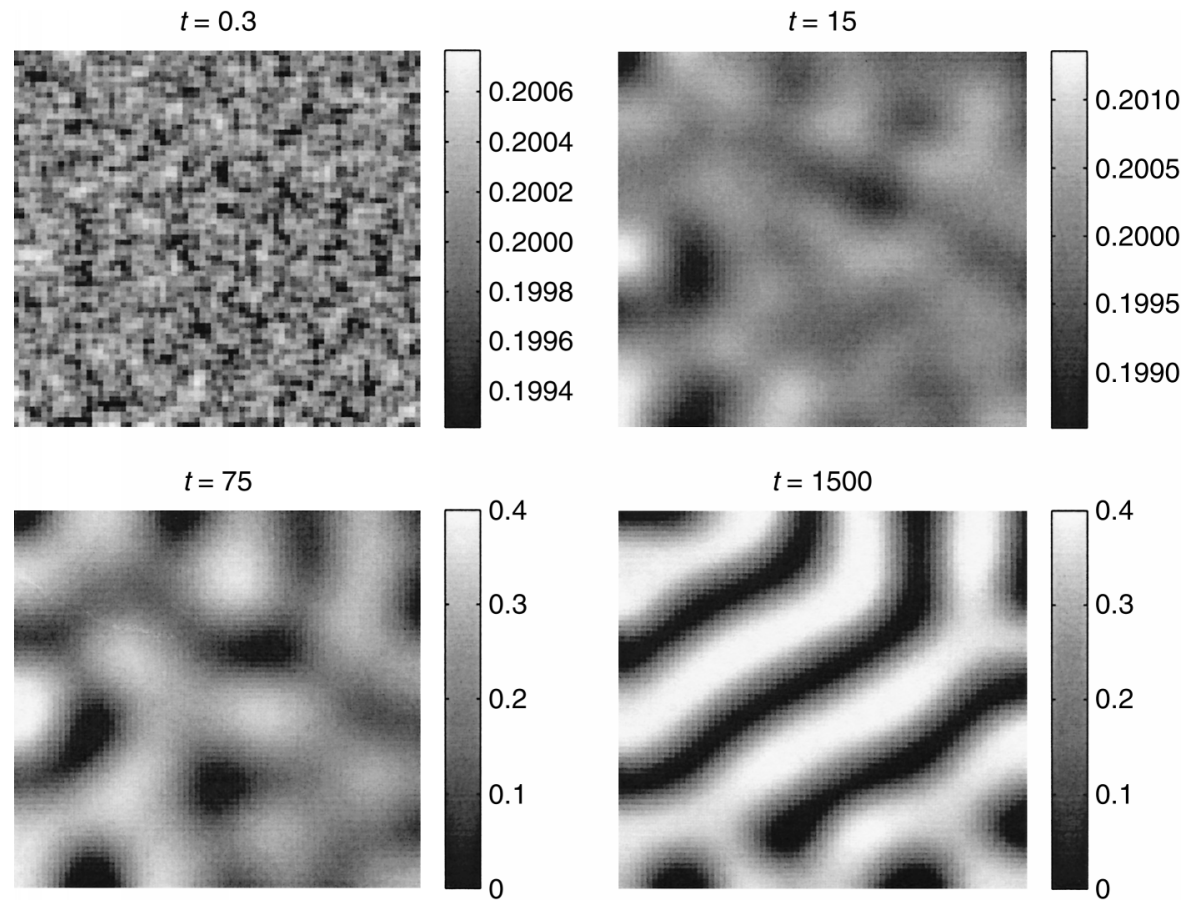

FIG. 1. Turing patterns in two dimensions. The numerical integration of the system described by Eqs. 11-12 has been performed from $t=0$ to $t=1500$ in a $75 \times 75$ lattice, using nonflux boundary conditions and initially (at $t=0)$ adding random and nonuniform small perturbations to the equilibrium values. The model parameters are $\beta=2, \varepsilon=0.5$, $\eta=0.6$, and $d=10$. Vertical bars on the right side of the panels show prey densities in dimensionless units. Only prey densities are plotted.

$$
\varepsilon(\beta-1)<\eta<\varepsilon \beta .
$$

Furthermore, it must be noticed that $G(\eta, 1)=g(\eta) /$ 2. As a consequence, for $d=1$, the conditions given by Inequalities 21 and 23 are not compatible. Since $d$ $=D_{P} / D_{N}$, predators must diffuse faster than prey in order to have diffusion instability (Okubo 1980).

Moreover, looking at the structure of the second-de-

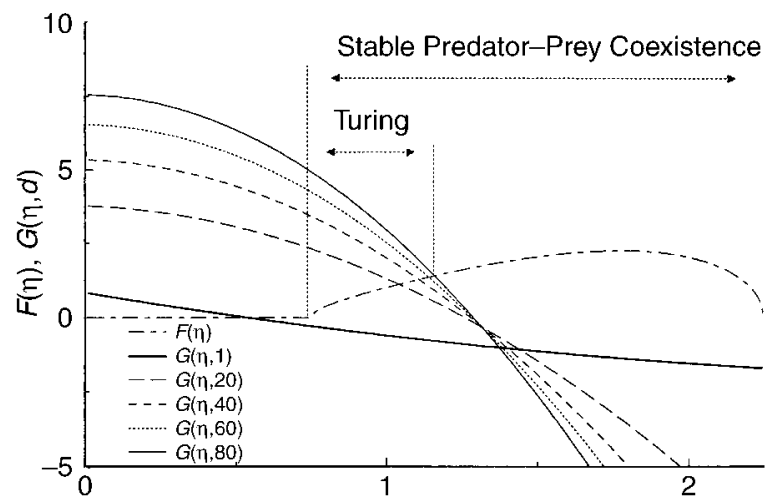

$\eta$, dimensionless predator mortality

FIG. 2. Bounding the Turing space: a graphic way to see where the three conditions given by Inequalities $21-23$ are met simultaneously for each pair of $\beta$ and $\varepsilon$ values (see Eqs. 13). The functions $g(\eta) / 2=G(\eta, 1)$ (Eq. 17), $F(\eta)$ (Eq. 19) $G(\eta, d)$ (Eq. 20) are plotted. In this example, $\beta=1.5$, and $\varepsilon=1.5$. It can be seen that there is a critical diffusion ratio, $d$, from which Turing instabilities emerge. The Turing space is indicated for diffusion ratio $d=80$. gree polynomial in $\eta$ (Eq. 20), it can be seen that increasing values of $d$ enhance the appearance of diffusion instabilities as long as $\beta>1$. Alternatively, if $\beta<1$, diffusion instabilities do not arise for increasing values of $d$. In Fig. 2 functions $G(\eta, d)$ and $F(\eta)$ are plotted and the other two conditions are represented for concrete values of $\beta$ and $\varepsilon$. The fulfillment of the three conditions define an $\eta$ range where Turing instabilities arise.

If a more general functional response (Beddington 1975) is assumed, the Turing space (Turing 1952) can only be computed numerically. A complete numerical exploration of the parameter space is presented in Fig 3 . It can be seen that Turing structures never arise when $\beta<1$. According to the definition of $\beta$ parameter (Eqs. $13)$, high predator attack rates $(b)$ in relation to prey growth rates $(r)$ should enhance the formation of Turing structures for a fixed degree of predator interference $(k)$. Moreover, the parameter subregion (white in Fig. 3 ), where diffusion instabilities take place, is particularly conspicuous for $\eta$ ranging from 1 to 50. The most realistic situations where Turing structures can arise are those where predator death rate and prey growth rate are similar $(\eta \approx 1)$. Assuming the conversion efficiency $(e)$ to be approximately constant, the greater the value of $\eta$, the higher both the degree of interference $(k)$ and the predator attack rate $(b)$ must be for Turing structures to arise.

Furthermore, the effects of a non-zero saturation constant ( $B$; Eq. 6) on parameter space can be seen in Fig. 4. It shows how introducing this saturation con- 

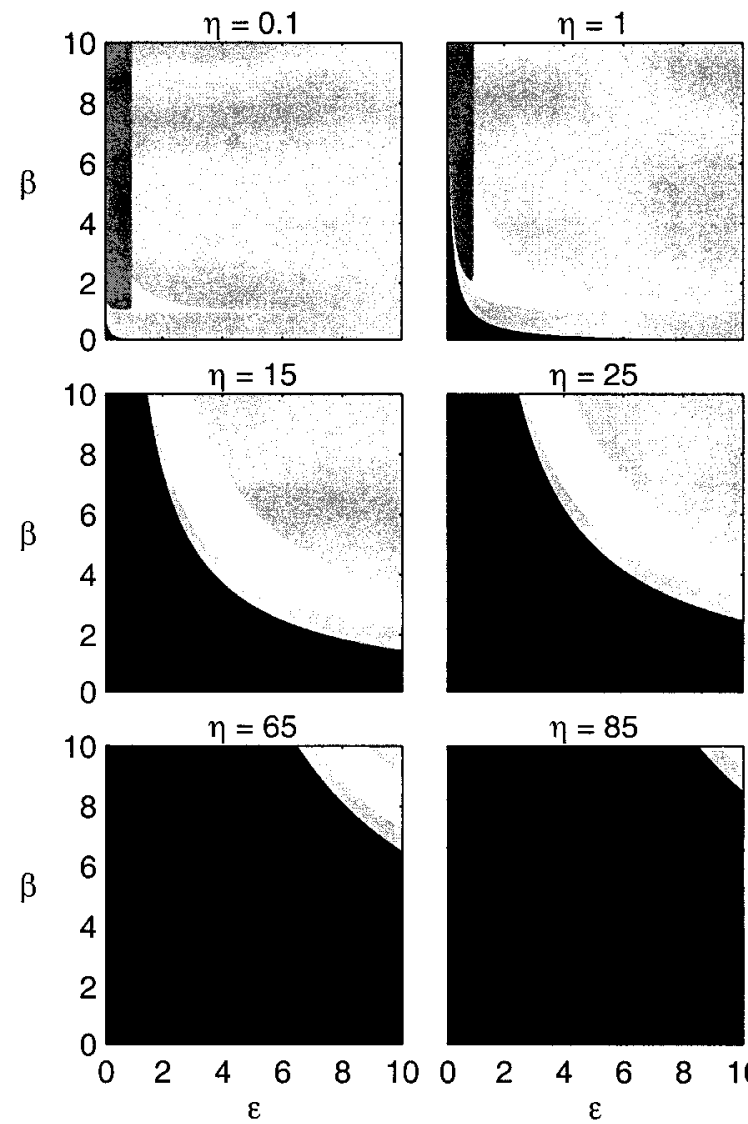

$\eta=25$

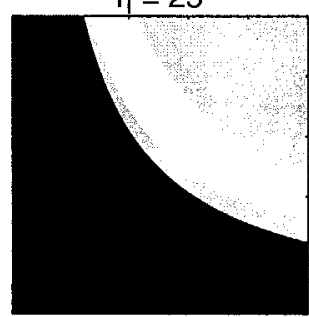

$\eta=85$
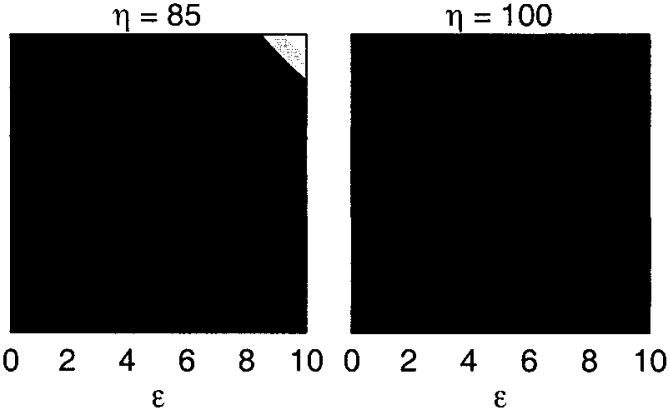

FIG. 3. Numerical exploration of the parameter space. For $B^{*}=0.001$, different domains can be distinguished in each plot for increasing values of the $\eta$ parameter. Here, $B^{*}$ is the dimensionless constant corresponding to the $B$ saturation constant that appears in Eq. 6. In fact, $B=N_{\max } \times B^{*}$. Turing space is shown in white, stable predator-prey coexistence in light grey, nonstable predator-prey coexistence in dark grey, and non-coexistence in black. The diffusion ratio is $d=100$; for definitions of $\beta, \eta$, and $\varepsilon$ see Eqs. 13 .

stant in predator functional response enhances both stability and the arising of Turing structures.

\section{DISCUSSION}

\section{A simple interpretation}

The basic idea of diffusion-driven instability in a reaction-diffusion system can be understood in terms of an activator-inhibitor system. The functioning of this mechanism is based on three points. First, a random increase of activator species should have a positive effect on the creation rate of both activator and inhibitor species. Second, an increment in inhibitor species should have a negative effect on formation rate of both species. Finally, inhibitor species must diffuse faster than activator species. Let us show that the reactiondiffusion predator-prey model (Eqs. 9 and 10), with a predator-dependent functional response and predators diffusing faster than prey, provides this mechanism.

Random fluctuations may cause a nonuniform prey density - the activator species. This elevated prey density has a positive effect both on prey and predator population growth rates. Look at per capita rates from Eqs. 9 and 10:

$$
\begin{aligned}
& \frac{1}{N} \frac{\partial N}{\partial T}=r\left(1-\frac{N}{N_{\max }}\right)-\frac{b P}{k P+N} \\
& \frac{1}{P} \frac{\partial P}{\partial T}=e \frac{b N}{k P+N}-\mu .
\end{aligned}
$$

Since Eq. 25 can be seen as a one-humped function of prey density $(N)$, growth prey rate can be increased by a higher local prey density at least in a range of parameter values. On the other hand, Eq. 26, i.e., predator numerical response, is an ever-increasing function of $N$. Higher prey densities always have a positive influence on predator growth.

Increased local predator density-the inhibitor species-has a negative effect in both per capita growth rates. The asymptotic behavior of the second term in Eq. 25 ensures a higher prey consumption at higher predator densities. The inhibitor mechanism on prey densities is then guaranteed. Predator interference in the functional response is responsible for the auto-inhibitor effect of increased predator densities on the growth of predator population (Eq. 26). Thus, as random fluctuations increase local prey density over its equilibrium value, prey population undergoes an accelerated growth. 


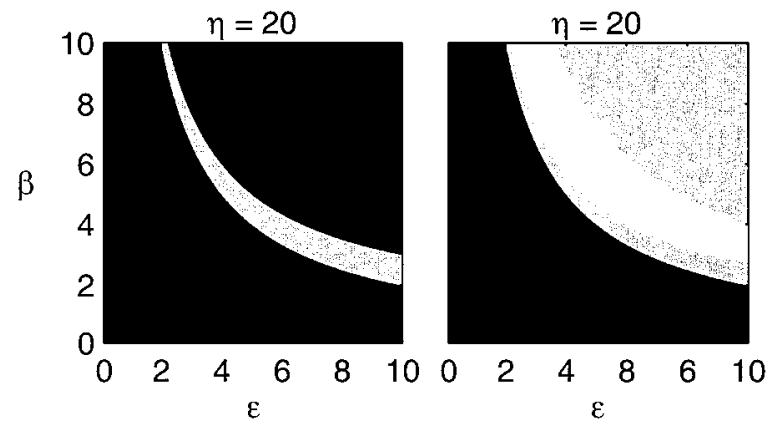

FIG. 4. Beddington effect. The parameter space is computed numerically for $B^{*}=0$ and $\eta=20$ (left) and $B^{*}=$ 0.001 and $\eta=20$ (right), where $B=N_{\max }$ (prey carrying capacity) $\times B^{*}$ (Beddington saturation constant) and $\eta=\mu$ [the per capita predator death rate] $/ r$ [prey growth rate]). The same predator-prey model, corresponding to the system described by Eqs. 9 and 10, can be compared either with a ratiodependent functional response or with a Beddington's functional response with regard to the occurrence of stable coexistence points (gray), Turing points (white), and non-coexistence points (black). The diffusion ratio is $d=100$.

Simultaneously, predator population also increases, but as predators diffuse faster than prey, they disperse away from the center of prey outbreaks. As a consequence, spatially uniform population distributions break down and two different spatial domains arise. In the center of the outbreaks prey growth rate keeps positive. As a consequence, prey population keeps increasing out of predator control. Thereafter, the proportion of prey to predators in those central areas increases. By contrast, on the border of prey outbreaks, the proportion of prey to predators becomes lower and lower. If relative diffusion $\left(D_{P} / D_{N}\right)$ is large enough, prey growth rate will reach negative values there and prey population will be driven by predators to a very low level in those regions. The final result is the formation of patches of high prey density surrounded by areas of low prey densities. Predators follow the same pattern, but, since they diffuse faster, their spatial distributions are smoother.

\section{Comparison with other reaction-diffusion models}

A great amount of previous work has been done looking for biological mechanisms of pattern formation through diffusion-driven instabilities (Okubo 1980). An early study calling attention to the fact that the diffusive instability can appear in simple predator-prey models was done by Segel and Jackson (1972). As an ecological example, they analyzed a Lotka-Volterra "exploiter-victim" system that incorporated two new features in relation to previous predator-prey models: (1) an autocatalytic effect on prey growth rate and (2) density-dependent mortality of predators. The same model was suggested later as a possible explanation for the origins of planktonic patchiness (Levin and Segel 1976). As a generalization of Segel and Jackson's model, Mimura and Murray (1978) and Conway (1984) considered other predator-prey models. Again density- dependent predator mortality and some kind of autocatalytic prey growth are two necessary conditions to obtain diffusion-driven instabilities. Without these peculiarities all these prey-dependent models cannot give rise to Turing structures (see Appendix).

Still some other mechanisms have been suggested to be involved in the generation of spatial patterns through trophic interaction. For instance, in a homogeneous environment, negative cross-diffusion (Jorné 1977, Chattopadhyah et al. 1996, Satulovsky 1996) has been identified as a factor increasing the possibility of spatiotemporal instabilities in Lotka-Volterra systems, although it can be considered quite rare from the ecological point of view "since it represents a suicidal tendency in the case of prey, and exploiters diffusing away from their victims in the case of predators" (Satulovsky 1996:381). More recently, environmental variability has received more attention. The interplay between environmental heterogeneity and trophic interaction (Benson et al. 1993, Pascual and Caswell 1997) can enhance pattern formation in an ecological context. Unlike other reaction-diffusion predator-prey models leading to Turing structures (Segel and Jackson 1972, Levin and Segel 1976, Mimura and Murray 1978, Conway 1984), notice that the activator-inhibitor mechanism of the model discussed here stems only from the predator dependency in the functional response. It is just the nature of predation that is solely responsible for the emergent spatial patterns.

However, one should have in mind that most organisms are neither simple diffusers nor move always randomly, but can display preferential directions of movement. This would add an advection term to the equations. Diffusion-advection-induced instabilities of spatially uniform distributions can occur for comparable diffusion coefficients, only the speed must be different (Rovinsky and Menzinger 1992, 1993). However, in these models the arising structures differ from Turing patterns. They are of the traveling-wave type. Obviously, nature is complex and several biotic and abiotic mechanisms are working at the same time to produce the observed spatial distributions. As usual, the crucial point is to assess the relative importance of each factor.

\section{CONCLUSION}

Keeping in mind how difficult empirical approaches are (but see Turchin et al. [1998] and references therein), our work immediately suggests a well-defined hypothesis that could be tested carrying out specific experiments: mutual interference between predators acts as an underlying mechanism able to produce nonuniform spatial distributions of predators and prey through diffusiondriven instability. Our theoretical approach suggests that the feeding strategy of predators (e.g., filtering, cruise or ambush hunting) may be the determining factor in producing Turing spatial patterns. Any strategy that involves interference between predators should enhance pattern formation. Furthermore, biological rate process-

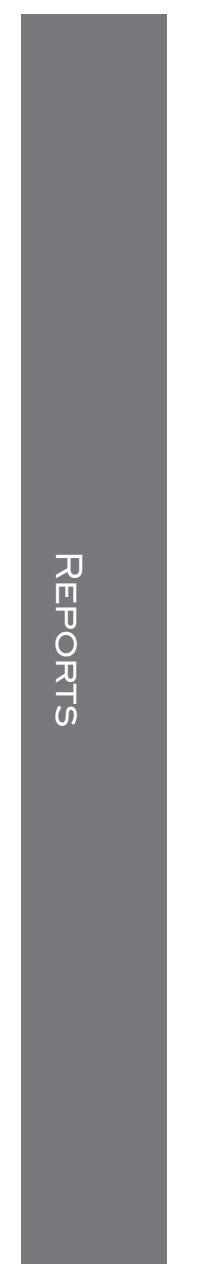


es (prey birth rate, predator death rate, predator attack rate) and size ratios affecting relative mobility can also be determining factors. A closer interpretation of the Turing space (Figs. 3 and 4) can unmask other generic constraints for predator-prey interactions.

Our main conclusion is that mutual interference between predators is one of the most convincing mechanisms for the spontaneous generation of patchiness through predator-prey trophic interaction in a homogeneous environment. Under the most simple assumptions, e.g., self-damping prey growth rate, linear conversion rule (Ginzburg 1998), and random death of predators, simple reaction-diffusion predator-prey models can give rise to spatial structures through diffusion instabilities as long as some degree of interference between predators is assumed. In the search for evidence in support of mutual interference between predators as a mechanism leading to formation of spatial structures, a lot of work is left to do. Only detailed data could support the model and its predictions. For instance, experiments testing particular hypotheses could be performed in meso- or microcosms to study microscale structure of planktonic spatial distributions. From our point of view, plankton pelagic communities are the best candidates in order to find empirical evidence with the least difficulty, although the mechanism should also operate in other aquatic and terrestrial systems. Therefore, we hope that the results presented here will suggest new empirical and theoretical studies.

\section{ACKNOWLEDGMENTS}

We would like to thank J. Bascompte, J. Saldaña, and R. V. Solé for valuable suggestions and interesting discussions. We thank two anonymous reviewers who helped to improve the manuscript. We also are indebted to P. Fernández and J. Piera for valuable computer assistance. This work has been supported by CIRIT grants 1999FI 00524 UPC APMARN to D. Alonso and 1997FI 00296 UB APMARN to F. Bartumeus from the Catalan autonomous government.

\section{Literature Cited}

Abrams, P. A. 1994. The fallacies of "ratio-dependent" predation. Ecology 75:1842-1850.

Abrams, P. A., and L. R. Ginzburg. 2000. The nature of predation: prey dependent, ratio dependent or neither? Trends in Ecology \& Evolution, 15:337-341.

Akçakaya, H. R., R. Arditi, and L. R. Ginzburg. 1995. Ratiodependent predation: an abstraction that works. Ecology 76:995-1004

Arditi, R., and L. R. Ginzburg. 1989. Coupling in predatorprey dynamics: ratio dependence. Journal of Theoretical Biology 139:311-326.

Beddington, J. R. 1975. Mutual interference between parasites or predators and its effect on searching efficiency. Journal of Animal Ecology 44:331-340.

Beddington, J., C. Free, and J. Lawton. 1976. Concepts of stability and resilience in predator-prey models. Journal of Animal Ecology 45:791-816.

Benson, D. L., J. A. Sherratt, and P. K. Maine. 1993. Dif- fusion driven instability in an inhomogeneous domain. Bulletin of Mathematical Biology 55:365-384.

Chattopadhyah, J., A. K. Sarkar, and P. K. Tapaswi. 1996. Effect of cross-diffusion on a diffusive prey-dependent system-a non linear analysis. Journal of Biological Systems 4:159-169.

Conway, E. D. 1984. Partial differential equations and dynamical systems. Springer-Verlag, Berlin, Germany.

Ginzburg, L. R. 1998. Assuming reproduction to be a function of consumption raises doubts about some popular predator-prey models. Journal of Animal Ecology 67:325-327.

Ginzburg, L. R., and H. R. Akçakaya. 1992. Consequences of ratio-dependent predation for steady-state properties of ecosystems. Ecology 73:1536-1543.

Holling, C. S. 1959. The components of predation as revealed by a study of small mammal predation of the European pine sawfly. Canadian Entomologist 91:293-320.

Holling, C. S. 1966. The functional response of invertebrate predators to prey density. Memoirs of the Entomological Society of Canada 48:1-86.

Huisman, G., and R. J. de Boer. 1997. A formal derivation of Beddington functional response. Journal of Theoretical Biology 185:389-400.

Jorné, J. 1977. The diffusive Lotka-Volterra system. Journal of Theoretical Biology 65:133-139.

Levin, S. A. 1992. The problem of pattern and scale in ecology. Ecology 73:1943-1967.

Levin, S. A., and L. A. Segel. 1976. Hypothesis for origin of planktonic patchiness. Nature 259:659.

Mimura, M., and J. D. Murray. 1978. On a diffusive preypredator model which exhibits patchiness. Journal of Theoretical Biology 75:249-262.

Murray, J. D. 1989. Mathematical biology. Springer-Verlag, Berlin, Germany.

Okubo, A. 1980. Diffusion and ecological problems: mathematical models. Springer-Verlag, Berling, Germany.

Pascual, M., and H. Caswell. 1997. Environmental heterogeneity and biological pattern in a chaotic predator-prey system. Journal of Theoretical Biology 185:1-13.

Rosen, G. 1977. Effects of diffusion on the stability of the equilibrium in multispecies communities. Bulletin of Mathematical Biology 39:373-383

Rovinsky, A. B., and M. Menzinger. 1992. Chemical instability induced by a differential flow. Physical Review Letters 69:1193-1196.

Rovinsky, A. B., and M. Menzinger. 1993. Self-organization induced by differential flow of activator and inhibitor. Physical Review Letters 70:778-781.

Ruxton, G. D., W. S. C. Gurney, and A. M. de Roos. 1992. Interference and generation cycles. Theoretical Population Biology 42:235-254.

Satulovsky, J. 1996. Lattice Lotka-Volterra models and crossdiffusion. Journal of Theoretical Biology 183:381-389.

Segel, L. A., and J. L. Jackson. 1972. Dissipative structure: an explanation and an ecological example. Journal of Theoretical Biology 37:545-559.

Turchin, P., J. Reeve, J. T. Cronin, and R. T. Wilkens. 1998. Spatial pattern formation in ecological systems: bridging theoretical and empirical approaches. Pages 199-208 in J. Bascompte and R. V. Solé, editors. Spatiotemporal dynamics in ecology. Springer and Landes Bioscience, Berlin, Germany.

Turing, A., 1952. The chemical basis of morphogenesis. Philosophycal Transactions of the Royal Society of London B 237:37-72.

Volterra, V. 1926. Variazioni e fluttuazioni del numero d'individui in specie animali conviventi. Memoria Academia Lincei 2:31-113.

\section{APPENDIX}

A demonstration of why the predator-prey models given by Eqs. 4 and 5, with prey-dependent functional responses, cannot give rise to Turing structures is available in ESA's Electronic Data Archive: Ecological Archives \#E083-001-A1. 